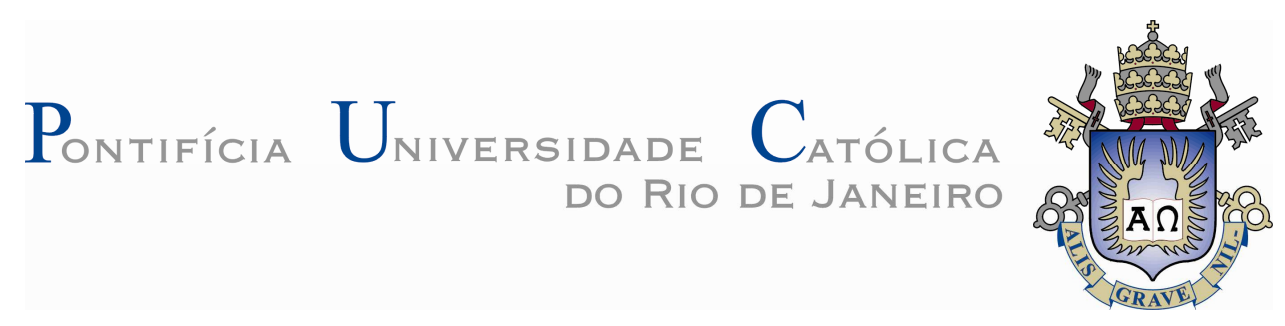

Elizabeth Suescún Monsalve

Construindo um Jogo Educacional com Modelagem Intencional Apoiado em Princípios de Transparência

Dissertação de Mestrado

Dissertação apresentada ao Programa de PósGraduação em Informática da PUC-Rio como requisito parcial para obtenção do título de Mestre em Informática.

Orientador: Prof. Julio Cesar Sampaio do Prado Leite

Rio de Janeiro

Março de 2010 


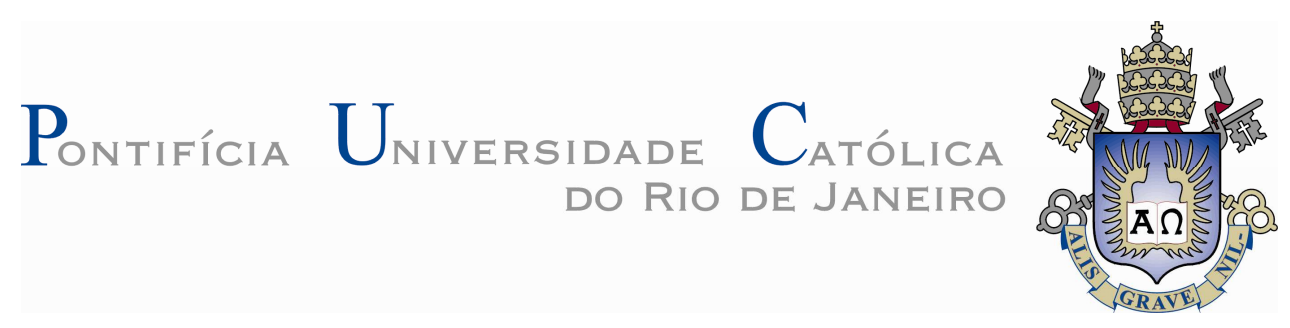

Elizabeth Suescún Monsalve

\title{
Construindo um Jogo Educacional com Modelagem Intencional Apoiado em Princípios de Transparência
}

\begin{abstract}
Dissertação apresentada como requisito parcial para obtenção do grau de Mestre pelo Programa de PósGraduação em Informática do Departamento de Informática do Centro Técnico e Científico da PUC-Rio. Aprovada pela Comissão Examinadora abaixo assinada.
\end{abstract}

Prof. Julio Cesar Sampaio do Prado Leite Orientador Departamento de Informática - PUC-Rio

Prof. Carlos José Pereira de Lucena Departamento de Informática - PUC-Rio

Profa Vera Maria Benjamim Werneck Departamento de Informática - UERJ-Rio

Prof. José Eugênio Leal Coordenador Setorial do Centro Técnico Científico - PUC-Rio 
Todos os direitos reservados. É proibida a reprodução total ou parcial do trabalho sem autorização da universidade, do autor e do orientador.

\section{Elizabeth Suescún Monsalve}

Graduou-se em Engenharia de Informática pelo PCJIC Institición Universitaria, em setembro de 2004. Área de interesse acadêmico: Engenharia de Software, mais especificamente em Engenharia de Requisitos.

Ficha Catalográfica

Monsalve, Elizabeth Suescún

Construindo um jogo educacional com modelagem intencional apoiado em princípios de transparência / Elizabeth Suescún Monsalve; orientador: Julio Cesar Sampaio do Prado Leite - 2010

$196 \mathrm{f} ; 30 \mathrm{~cm}$

Dissertação (Mestrado em Informática) - Pontifícia Universidade Católica do Rio de Janeiro, Rio de Janeiro, 2010.

Inclui bibliografia.

1. Informática - Teses. 2. Engenharia de Requisitos. 3. Jogos Educacionais. 4. Framework $i *$. 5. Modelagem Intencional. 6. Transparência de Software I. Leite, Julio César Sampaio do Prado. II. Pontifícia Universidade Católica do Rio de Janeiro. Departamento de Informática. III. Título.

CDD: 004 
Este trabalho é dedicado a minha Mãe e Carlos com todo meu amor. 


\section{Agradecimentos}

Primeiramente, agradeço a Deus.

Agradeço ao órgão de financiamento CAPES pela bolsa oferecida a qual viabilizou esta pesquisa.

Ao meu orientador, professor Julio Cesar Sampaio do Prado Leite, pela confiança depositada em mim, pela paciência e por todas as orientações durante este trabalho.

À professora Vera Maria Benjamim Werneck, pelos aportes e colaboração durante este trabalho.

À minha querida mãe, pela fé, a confiança e por esse amor incondicional.

A Zammis pela alegria que cada dia da a minha vida.

A Giba por todo esse amor y carinho que me entregou, e que agora desde o céu continua vivo.

Aos amigos do grupo de Engenharia de Requisitos e aos alunos voluntários, pelas valiosas contribuições a este trabalho.

A meus amigos Edy, Sandra, Kátia, Paola, Andréia, Marcio, Antonio, Juan Pablo e Bruno que longe ou perto sempre estiveram me apoiando e me acompanhando. 


\section{Resumo}

Monsalve, Elizabeth Suescún; Leite, Julio Cesar Sampaio do Prado. Construindo um Jogo Educacional com Modelagem Intencional Apoiado em Princípios de Transparência. Rio de Janeiro, 2010. 196p. Dissertação de Mestrado - Departamento de Informática, Pontifícia Universidade Católica do Rio de Janeiro.

Jogos educacionais vêm sendo propostos no ensino de ciências da computação, e também no ensino de a engenharia de software. Neste trabalho, apresentamos uma abordagem de modelagem intencional apoiada em conceitos de transparência para a implementação do jogo educacional SimulES. SimulES é um jogo para apoiar o ensino de engenharia de software. A abordagem é inovadora neste contexto. Acreditamos que a modelagem intencional é pertinente para modelar jogos, já que ela permite representar a interação e colaboração entre os atores, além de apoiar conceitos de transparência. Essa modelagem foi usada no desenvolvimento do software SimulES-W que implementa o jogo num ambiente Web.

\section{Palavras-chave}

Engenharia de Requisitos; Jogos Educacionais; Framework $i$; Modelado Intencional; Transparência de Software 


\section{Abstract}

Monsalve, Elizabeth Suescún; Leite, Julio Cesar Sampaio do Prado (Advisor). Building an Educational Game with Intentional Modeling Supported with Principles of Transparency. Rio de Janeiro, 2010. 196p. MSc. Dissertation - Departamento de Informática, Pontifícia Universidade Católica do Rio de Janeiro.

Educational games have been proposed for teaching computer science, and software engineering as well. This work presents an approach for intentional modeling supported by concepts of transparency towards the implementation of the educational game SimulES. SimulES is a game for helping software engineering teaching. The approach is innovative in that context. We believe that intentional modeling is akin to game modeling, since it allows us to represent the interaction and collaboration among the actors as well concepts of transparency. The intentional model we produced was used to develop the software that implements SimulES-W, a Web based version of the game.

\section{Keywords}

Requirements Engineering; Educational Game; Framework $I^{*}$; Intentional Modeling; Software Transparency 


\section{Sumário}

1 Introdução 17

1.1. Motivação 17

1.2. Objetivos 18

1.3. Conceitos 18

1.3.1. Léxico Ampliado da Linguagem (LAL) 18

1.3.2. Cenários 19

1.3.3. Visão Geral do Framework $i^{*} \quad 20$

1.4. Organização da Dissertação 31

2 Jogos Educacionais 32

2.1. Visão Geral de Jogos Educacionais 32

2.1.1. O Jogo Problems and Programmers (PnP) 32

2.1.2. O Jogo SESAM 34

2.1.3. O Jogo SimVBSE

2.1.4. O Jogo SimSE 37

2.1.5. O Jogo Planager 39

2.1.6. O Jogo Scrumming 40

2.1.7. O Jogo X-MED v1.0 41

2.1.8. O Jogo SimulES 42

2.2. Resumo das Características dos Jogos 43

2.3. Considerações Sobre Jogos Educacionais 43

3 Procurando Elementos de Evolução do SimulES 45

3.1. Contextualização 46

3.1.1. O SimulES 46

3.1.2. Técnicas Utilizadas para Identificar Elementos de Evolução para o SimulES 54

3.1.3. Usando as Técnicas de Elicitação para a Evolução do SimulES 55 
4 Uso das Representações $\quad 64$

4.1. Contextualização 64

4.2. Representação de Requisitos em Linguagem Natural 65

4.2.1. Léxico Ampliado da Linguagem (LAL) 65

4.2.2. Cenários 66

$\begin{array}{ll}\text { 4.2.3. C\&L } & 67\end{array}$

4.3. Representação Intencional dos Requisitos 67

4.3.1. Framework $i^{*} \quad 68$

4.3.2. O Método $E R i^{*} \mathrm{C} \quad 68$

4.3.3. Modelagem Intencional do SimulES 70

4.4. Análise das Representações de Requisitos Usadas 80

5 Trabalhos Relacionados $\quad 81$

5.1. Modelagem Intencional Casos Práticos 81

5.1.1. Estendendo Tropos para uma Implementação em Prolog: um Estudo de Caso Usando o Problema do Agente Coletor de Alimentos (FCAP) [49] 81

5.1.2. "Tropos: uma Metodologia de Desenvolvimento de Software Orientada a Agentes" [50] 84

5.1.3. "Uma Abordagem para Modelagem Intencional de Avaliação de Riscos de Segurança em Web Services" [51] 89

5.2. Transparência de Software 93

5.2.1. "Uma Análise Inicial sobre como Transparência Software e Confiança se Influenciam Mutuamente" [52] 94

5.2.2. Transparência e Pureza do Software [54] 94

5.2.3. Explorando as Características de $i^{*}$ que

Apóiem a Transparência do Software [53] 96

5.3. Análise dos Trabalhos Relacionados 97

6 Construindo o SimulES-W 100

6.1. Background 100

6.2. Arquitetura do SimulES-W e Tecnologias Utilizadas 102

6.3. Método Usado na Construção do SimulES -W 103 
6.3.1. Primeira Etapa 103

6.3.2. Segunda Etapa 105

6.4. Incorporando Elementos em cada uma das Camadas 106

6.4.1. Identificar as SDsituations Principais 107

6.4.2. SDsituations Auxiliares 126

6.4.3. Descrição das SDsituations do Sistema Através de Cenários 133

6.4.4. Refinamento das SDsituations e dos Cenários 146

6.5. Cenários do Sistema 155

6.5.1. Cenários da Camada de Controle 155

6.5.2. Cenários da Camada de Modelo 160

6.5.3. Cenários da Camada de Visão 164

6.6. Operacionalização das SDsituations 164

6.6.1. Frameworks de Desenvolvimento Web 165

6.6.2. Ferramentas de Desenvolvimento 165

6.6.3. Desenvolvimento do SimulES-W 166

6.7. Rastreabilidade em SimulES-W 182

6.8. Avaliação dos Atributos da Transparência 184

7 Conclusão 187

7.1. Comparação com Trabalhos Relacionados 189

7.2. Dificuldades Encontradas 189

7.3. Trabalhos Futuros 190

8 Referências Bibliográficas 192 


\section{Lista de figuras}

Figura 1 - Ilustração da dependência entre atores utilizada em modelos SD. 23

Figura 2 - Decomposição de tarefas. $\quad 24$

Figura 3 - Elos meios-fim. 25

Figura 4 - Ilustração de um modelo SA [11]. 28

Figura 5 - Variantes de interdependência lógica das SDsituations [11]. 29

Figura 6 - Ilustração de um painel de intencionalidade adaptado de [11]. 31

Figura 7 - Tela principal do jogo SESAM [2]. 35

Figura 8 - Fluxo do jogo SimVBSE [3]. 37

Figura 9 - Tela principal do jogo SimSE tomado de [13]. 38

Figura 10 - SADT para SimulES-W. 45

Figura 11 - Dinâmica do jogo SimulES v 2.0. 47

Figura 12 - Exemplo de cartão de projeto SimulES v 2.0 [4]. 49

Figura 13 - Tabuleiro individual SimulES v 2.0 [4]. 49

Figura 14 - Exemplo de uma carta problema do jogo SimulES v 2.0 [4]. $\quad 50$

Figura 15 - Exemplo de carta conceito do jogo SimulES v 2.0 [4]. 51

Figura 16 - Exemplo de cartas de engenheiros de software SimulES v 2.0 [4]. 51

Figura 17 - Artefatos (a) brancos com ou sem erro e

(b) cinzas com ou sem erro SimulES v 2.0 [4]. 52

Figura 18 - Tabuleiro principal de SimulES v 2.0 [4]. 53

Figura 19 - Exemplo de cenário SimulES v 2.0 [4]. 55

Figura 20 - Representação gráfica dos resultados das questões fechadas. $\quad 58$

Figura 21 - Representação no CEL do léxico

usando a palavra chave "Jogador" v 2.0 [4]. 65

Figura 22 - Representação no CEL do léxico usando a palavra chave

“Jogador" v 3.0 [44].

Figura 23 - Exemplo do comportamento dos cenários do jogo v 2.0 [4]. 66

Figura 24 - Interface do C\&L usado para modelar elemento do jogo SimulES. 67

Figura 25 - Visão geral do método ERi*c [11]. 69

Figura 26 - Exemplo de símbolo do LAL para SimulES v 3.0 [44]. 71

Figura 27 - Símbolos tipo sujeito v 3.0 [44]. 71 
Figura 28 - Diagrama de SDsituations do SimulES v 3.0 [44]. 75

Figura 29 - modelo SA para SimulES v 3.0 [44]. 76

Figura 30 - Diagrama IP - Joga Rodada de Início do SimulES v 3.0

$\begin{array}{ll}\text { adaptado de [44]. } & 77\end{array}$

Figura 31 - Modelo SD - Joga Rodada de Início do SimulES v 3.0 [44]. 78

Figura 32 - Modelo SR - Joga Rodada de Início do SimulES v 3.0 [44]. 79

Figura 33 - Descrição através de cenários de SDsituation

Inspeção de Artefato do Simules v 3.0 [44]. 80

Figura 34 - Identificação de atores genéricos para FCAP [49]. 82

Figura 35 - Descrevendo papéis de agentes em FCAP [49]. 83

Figura 36 - Decomposição de cada agente FCAP [49]. 83

Figura 37 - Diagrama de atores, modelando os stakeholders

do projeto eCulture [50].

Figura 38 - Diagrama de metas para Cidadão e Visitante.

Observe se a meta e plano de decomposição,

a análise meios-fim e a contribuição positiva à meta flexível [50]. 86

Figura 39 - Segmento do diagrama de atores incluindo PAT

e o sistema eCulture e o diagrama de metas do sistema eCulture [50]. $\quad 87$

Figura 40 - Ambiente de desenvolvimento JACK para projeto eCulture. $\quad 88$

Figura 41 - Diagrama SD para o Web Service baseado em

cartões de pagamento.

90

Figura 42 - Tipos de atores do Web Service baseado em

cartões de pagamento [51].

91

Figura 43 - Diagrama SR para o Web Service baseado em

cartões de pagamento [51].

92

Figura 44 - Representação de ataques para o Web Service baseado em cartões de pagamento [51].

Figura 45 - Escada da Transparência tomada de [53].

96

Figura 46 - Arquitetura projetada para o SimulES-W.

Figura 47 - Primeira versão do modelo de classes para SimulES-W.

104

Figura 48 - Símbolo SimulES analisado para sua conversão

a classe do sistema.

Figura 49 - SDSituations candidata a página no SimulES-W. 
Figura 50 - Modelo de navegabilidade da aplicação Web para o SimulES-W. 106

Figura 51 - Diagrama SDsituations refinado para o SimulES-W. 107

Figura 52 - Modelo SA SimulES-W refinado. 107

Figura 53 - Modelo SD - Joga Rodada de Início. 108

Figura 54 - Modelo SR - Joga Rodada de Início. 109

Figura 55 - Modelo SD - Joga Rodada de Ações. 110

Figura 56 - Modelo SR - Joga Rodada de Ações. 111

Figura 57 - Modelo SD - Construção de Artefato. 112

Figura 58 - Modelo SD - Construção de Artefato. 113

Figura 59 - Modelo SD - Inspeção de Artefato. 114

Figura 60 - Modelo SD - Inspeção de Artefato. 115

Figura 61 - Modelo SD - Correção de Artefato. 116

Figura 62 - Modelo SD - Correção de Artefato. 117

Figura 63 - Modelo SD - Joga Rodada de Conceitos. 118

Figura 64 - Modelo SR - Joga Rodada de Conceitos. 119

Figura 65 - Modelo SD - Tratamento de Problema. 120

Figura 66 - Modelo SR - Tratamento de Problema. 121

Figura 67 - Modelo SD - Submissão de Produto. 122

Figura 68 - Modelo SR - Submissão de Produto. 123

Figura 69 - Modelo SD - Integração de Artefato em Módulo. 124

Figura 70 - Modelo SR - Integração de Artefato em Módulo. 125

Figura 71 - Modelo SD - Apresentar Dinâmica do Jogo. 127

Figura 72 - Modelo SR - Apresentar Dinâmica do Jogo. 128

Figura 73 - Modelo SD - Gestão de Material de Apoio. 129

Figura 74 - Modelo SR - Gestão de Material de Apoio. 130

Figura 75 - Modelo SD - Gestão do Jogo. 131

Figura 76 - Modelo SR - Gestão do Jogo. 132

Figura 77 - Modelo SD - Apresentar Dinâmica do Jogo

com atributos da Transparência.

Figura 78 - Modelo SR - Apresentar Dinâmica do Jogo

com atributos da Transparência.

Figura 79 - MVC para aplicações Web [63]. 164

Figura 80 - Tela da SDsituation Apresentar Dinâmica do Jogo. 167 
Figura 81 - Parte do código da SDsituation Apresentar Dinâmica do Jogo. 168

Figura 82 - Tela da SDsituation Joga Rodada de Inicio. 168

Figura 83 - Tela da SDsituation Joga Rodada de Ações. 169

Figura 84 - Tela da SDsituation Construção de Artefatos. 170

Figura 85 - Tela da SDsituation Inspeção de Artefato. 170

Figura 86 - Tela da SDsituation Correção de Artefato. 171

Figura 87 - Tela da SDsituation Joga Rodada de Conceitos

ações sobre os Engenheiros de Software. 171

Figura 88 - Tela da SDsituation Joga Rodada de Conceitos ação

de Descartar Cartas.

Figura 89 - Tela da SDsituation Joga Rodada de Conceitos ação de Submeter Problema.

Figura 90 - Tela da SDsituation Tratamento de Problema, problemas por jogador.

Figura 91 - Tela da SDsituation Tratamento de Problema, tratamento com Carta Conceito.

Figura 92 - Tela da SDsituation Tratamento de Problema, tratamento com penalidade.

Figura 93 - Tela da SDsituation Tratamento de Problema, aceitação de tratamento problema pelos jogadores.

Figura 94 - Tela das SDsituations

Integração de Artefatos no Módulo e Submissão de Produto.

175

Figura 95 - Tela da SDsituation Gestão de Material de Apoio, criar material de apoio.

Figura 96 - Tela da SDsituation Gestão de Material de Apoio, operações sobre Cartas Conceito e Cartas Problema. 176

Figura 97 - Tela da SDsituation Gestão do Jogo. 176

Figura 98 - Operacionalização dos cenários da camada do modelo. 178

Figura 99 - Parte de código do cenário Tabuleiro Individual na camada modelo.

Figura 100 - Operacionalização dos cenários da camada do controle.

Figura 101 - Parte de código do cenário controlador de rondas da camada controle. 
Figura 102 - Segmento da SDsituation Dinâmica do Jogo para ilustrar Rastreabilidade. 


\section{Lista de tabelas}

Tabela 1 - Estrutura de cenário adaptada de [59] 19

Tabela 2 - Resumo das características dos jogos. 43

Tabela 3 - Questionário SimulES aplicado a estudantes da UERJ. 56

Tabela 4 - Regras gerais para definição de símbolos [22]. 70

Tabela 5 - Template preenchido com metas dos símbolos do tipo sujeito [44]. 72

Tabela 6 - Template com metas concretas do símbolo do tipo objeto [44]. 73

Tabela 7 - Template com metas flexíveis do símbolo do tipo objeto [44]. 73

Tabela 8 - Template com metas do símbolo do tipo verbo [44]. 73

Tabela 9 - Template com metas do símbolo do tipo estado [44]. 73

Tabela 10 - Template para agrupar e organizar metas por ator cronologicamente [44].

Tabela 11 - SimulES-W e os termos da Transparência. 184 